\title{
El editor de mapas conceptuales DigiDocMap
}

\author{
Cristòfol Rovira Fontanals *
}

Artículo recibido:

30 de noviembre de 2005.

Artículo aceptado:

2 de enero de 2006.

\section{RESUMEN:}

DigiDocMap es un editor freeware y open source para la creación de mapas conceptuales. Es el primer resultado de un proyecto de investigación orientado a la generación de herramientas para representar el conocimiento. Se han desarrollado tres versiones de este editor desde el año 2002. En el artículo se analizan las prestaciones de cada una de ellas y las razones que motivaron la incorporación de los principales cambios.

Palabras clave: Mapa conceptual; Organización de la información; Representación del conocimiento; Visualización de la información; Topic Maps.

* Universidad Pompeu Fabra, cristofol.rovira@upf.edu INVESTIGACIÓN BIBLIOTECOLÓGICA, Vol. 21, Núm. 42, enero/junio, 2007, México,
ISSN: 0187-358X. pp. 65-78 


\section{ABSTRACT:}

\section{The editor of conceptual maps, DigiDocMap Cristòfol Rovira Fontanals}

DigiDocMap is freeware editor and open source for the creation of conceptual maps. It is the first product of a research project intended to generate tools for knowledge representation. Three versions of this editor have been created since 2002. The paper analyses each one of these and the reasons which motivated incorporation of the principal modifications.

Keywords: Conceptual map; Organization of information; Knowledge representation; Visualization of information; Topic maps.

\section{INTRODUCCIÓN}

L a representación gráfica de ideas o conceptos tiene un especial interés en el entorno de la organización de la información. Los esquemas gráficos facilitan la comprensión y la memorización. Este interés es todavía mayor si los contenidos se encuentran en soporte digital y en formato Web. En este entorno las representaciones gráficas pueden además facilitar la navegación. Los mapas de navegación clásicos muestran la arquitectura de las páginas de una sede Web y permiten orientar la navegación a través de esta estructura "física", pero son incapaces de representar la estructura conceptual de los contendidos de esas páginas. Para proporcionar una orientación basada en la estructura semántica de los contenidos de una Web es necesario usar mapas de conceptos, en especial cuando estos materiales digitales son materiales didácticos.

De entre todos los sistemas de representación gráfica de conceptos, los mapas conceptuales (Novak 1982, 1984, 1988) son las herramientas que permiten representar la estructura conceptual de un contenido didáctico de forma más simple, flexible y compacta. Es estos momentos hay una multitud de herramientas que permiten la creación y edición de mapas conceptuales para ser implementados en una página Web. Estas herramienta no son compatibles entre ellas ni con los estándares más extendidos como el Topic Maps. En este artículo se describe el desarrollo de un editor de uso libre y Open source para la creación y edición de mapas conceptuales que permite la exportación a XML Topic Maps.(XML, Extensible Markup Languaje, Lenguaje Extensible de Marcado LEM). 
Los resultados aquí presentados forman parte de un proyecto de investigación más amplio cuyo objetivo es desarrollar de métodos, procesos y herramientas en el contexto de la Web Semántica. Es un proyecto financiado por el Ministerio de Educación y Cultura (Plan Nacional I+D+I referencia HUM2004-03162/FILO).

\section{NOVAK Y LOS ORÍGENES DE LOS MAPAS CONCEPTUALES}

El origen de los mapas conceptuales hay que situarlo en los trabajos de Joseph D. Novak a mediados de la década de los sesenta en el contexto de un proyecto de investigación en psicología del aprendizaje. Novak (1982, 1984, 1988) investigaba el aprendizaje en niños basándose en las teorías de Ausubel (1989) y creó los mapas conceptuales como instrumento para hacer visible el tipo de aprendizaje adquirido. Novak no pensó en ningún momento en Internet, entre otras razones porque ésta no existía cuando creó los mapas conceptuales. Después se ha descubierto la gran utilidad que puede tener este instrumento como herramienta de acceso a la información en entornos digitales al añadirles enlaces hipertextuales a los conceptos de los mapas.

Un mapa conceptual, según la propuesta de Novak, es un conjunto de proposiciones sobre un determinado tema, ordenadas en forma de árbol. En los nodos de este árbol se colocan los conceptos, y en las conexiones entre estos nodos irían situadas frases de enlace que expresan la relación entre los conceptos conectados. Normalmente los conceptos son la parte sustantiva de las oraciones y los verbos y preposiciones constituyen las frases de enlace. Siempre hay un concepto inicial (raíz) a partir del cual se desarrolla el árbol de relaciones. Los conceptos se suelen representar dentro de cuadrados u óvalos, y las frases de enlace actúan como etiquetas de las líneas que unen dos o más de estos cuadrados de conceptos.

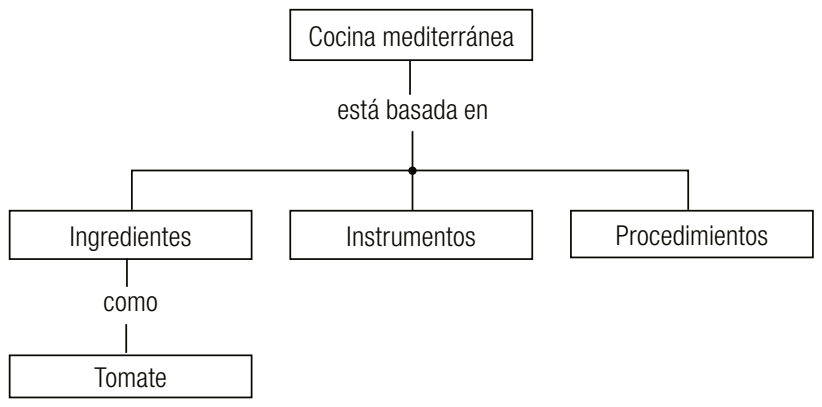

Fig. 1. Ejemplo de mapa creado con DigiDocMap 3 
Tabla 1. Los mapas conceptuales según Novak

\begin{tabular}{|l|l|}
\hline \multicolumn{1}{|c|}{ Característica } & \multicolumn{1}{c|}{ Mapas conceptuales (Novak ) } \\
\hline ¿Qué es? & $\begin{array}{l}\text { Una técnica para estructurar el conocimiento en conceptos y } \\
\text { frases de conexión para luego representarlo gráficamente }\end{array}$ \\
\hline Origen & $\begin{array}{l}\text { Investigaciones de Novak sobre psicología del aprendizaje, } \\
\text { basadas en las teorías de Ausubel (1989) }\end{array}$ \\
\hline Funciones & Facilitar y mejorar el aprendizaje \\
\hline Objetivos & $\begin{array}{l}\text { Representar la estructura de conocimientos de un estudiante } \\
\text { para conocer qué tipo de aprendizaje ha adquirido }\end{array}$ \\
\hline Fecha de origen & $\begin{array}{l}1984 \text { año de publicación de Novak, J. D.; D. B. Gowin. } \\
\text { Learning How to Learn. New York, Cambridge: Cambridge }\end{array}$ \\
\hline Partes constituyentes básicas & University Press, 1984 \\
\hline Aplicaciones & $\begin{array}{l}\text { Conceptos } \\
\text { Frases de enlace }\end{array}$ \\
\hline Estructura de la información implícita & Enlaces entre los conceptos \\
\hline Admite referencias cruzadas & Formación \\
\hline Orientación del mapa & Representación del conocimiento \\
\hline Necesariamente existe un concepto raíz & Sí \\
\hline Requiere representación gráfica & Síncipalmente de arriba a abajo \\
\hline Admite multijerarquía & Sí \\
\hline Admite enlaces desde los conceptos/topics & Sí \\
\hline a recursos externos & No (en su formulación inicial) \\
\hline Admite relación con dirección & Sí \\
\hline Admite relación sin dirección & Sí \\
\hline Admite relación bidireccional & Sí \\
\hline
\end{tabular}

\section{El editor de mapas conceptuales DigiDocMap}

Existen multitud de aplicaciones para la edición de mapas conceptuales. La mayoría son comerciales, algunas son de uso libre. En general cumplen con los requerimientos de la propuesta del mapa de Novak.

Editores comerciales de mapas conceptuales:

- Axon Idea Processor

http://web.singnet.com.sg/ axon2000/index.htm

- ConceptDraw http://www.conceptdraw.com/en/products/mindmap/main.php

- Decision Explorer http://www.banxia.com/demain.html 
- DigiDocMap http://www.mapasconceptuales.com

- Inspiration http://www.inspiration.com/

- Knowledge Manager http://www.conceptmaps.it/default-esp.htm

- Mind Graph eBook http://www.mind-graph.net/mind-graph.htm

- MindGenius http://www.mindgenius.com/

- MindMan http://usuarios.iponet.es/casinada/32mind.htm

- MindManager http://www.mindjet.com/eu/

- MindMapper http://www.mindmapperusa.com/

- NovaMind http://www.nova-mind.com/

- OmniGraffle http://www.omnigroup.com/applications/omnigraffle/

- PersonalBrain http://www.thebrain.com/

- Smartdraw http://www.smartdraw.com

- VisiMap http://usuarios.iponet.es/casinada/21vmap.htm

- VisualMind http://www.visual-mind.com/

Editores de uso libre freeware de mapas conceptuales:

- CmapTools http://cmap.ihmc.us/

- DigiDocMap http://www.mapasconceptuales.com

- FreeMind http://freemind.sourceforge.net/wiki/index.php/Main_Page

- Kdissert http://freehackers.org/ tnagy/kdissert/ 
- MyMindSimple

http://www.sebastian-krauss.de/software/

- Shared Space

http://www.shared-space.net/

- ThinkGraph

http://www.thinkgraph.com/

- VYM

http://www.insilmaril.de/vym/

DigiDocMap es una aplicación para la creación de mapas conceptuales, que cumple plenamente con los requerimientos de la propuesta de Novak. Es una aplicación de uso libre (freeware) y Open source. A diferencia de los otros editores de uso libre DigiDocMap también está disponible para usarse en línea, sin instalaciones previas en la dirección:

http://www.mapasconceptuales.com.

Los usuarios usan el editor entrando la información sobre conceptos y frases de enlace en formularios en una típica interfaz de usuario creada en una página Web. El editor genera una página HTML (Hiper Text Markup Language, Lenguaje de Marcado para Hipertexto) que contiene el mapa en forma gráfica. En el WebLog profesional "Servicio de Alerta" se pueden consultar algunos ejemplos de mapas conceptuales creados con DigiDocMap http://docdigital.typepad.com/

DigiDocMap forma parte de un conjunto de herramientas desarrolladas para la Maestría en Línea (Master Online) en Documentación Digital, que se ofrecen como herramientas de uso libre:

- DigiDocMap editor de mapas conceptuales

- DigiDocMenu editor de menús desplegables

- DigiDocIndex indexador y generador de buscadores locales

Estas herramientas están disponibles en:

http://www.documentaciondigital.org/master/laboratorio.htm

La motivación para iniciar el proyecto de la creación de una aplicación para editar y crear mapas conceptuales hay que situarla en los primeros años de docencia de la Maestría en Línea (Master Online) en Documentación Digital, (Rovira, 2001). En el diseño de los materiales didácticos para este curso se detectó la necesidad de incluir elementos adicionales que permitieran la navegación a través de rutas guiadas por criterios semánticos. La arquitectura de la información del aula digital del Master potenciaba la navegación a través de enlaces de tipo estructural (como por ejemplo, del curso al módulo, 
y del módulo a la unidad didáctica), pero no aparecían de manera explícita conexiones hipertextuales entre los contenidos de dos unidades didácticas que tuvieran elementos complementarios.

Al principio topamos con algunas dificultades importantes para crear este tipo de enlaces transversales. Cada unidad didáctica se encargó (y se le encarga) a un especialista que normalmente no conoce con suficiente profundidad los contenidos de la Maestría (Master) como para enlazar sus contenidos con otras unidades. Por otro lado, es delicado y engorroso negociar la inclusión de enlaces a los documentos originales recibidos. Nuestra decisión fue encargarle esta labor a la coordinadora de la Maestría (Master). Optamos por no incorporar estos enlaces en los artículos, sino que elaboramos un instrumento adicional, en una página independiente, para sintetizar de manera compacta estas conexiones semánticas entre los contenidos de diferentes unidades didácticas del curso. Este instrumento no es otro que los mapas conceptuales.

Para incorporar los mapas en el aula digital del curso, era necesario contar con una aplicación que permitiera crear y editar los mapas en formato HTML. Además era imprescindible que los conceptos incluidos en el mapa pudieran tener un listado de enlaces asociado ya que éste era nuestro principal objetivo. En el momento que iniciamos este proyecto no había ninguna aplicación que cumpliera con estos requerimientos. En los siguientes años, algunos editores disponibles en la Red añadieron la prestación de listados de enlaces hipertextuales asociados a los conceptos.

Aunque la motivación inicial fue, como decimos, añadir rutas alternativas de navegación en el aula digital de la Maestría (Master), luego se hicieron evidentes nuevas prestaciones que hacen de los mapas conceptuales una herramienta de gran utilidad en entornos formativos. En especial cabe destacar su capacidad para mostrar de forma resumida los contenidos didácticos y su extraordinaria eficacia cuando se usa como actividad de aprendizaje. Es muy ilustrativo observar un mapa creado por un especialista, pero es más interesante e instructivo que uno mismo haga su propio mapa. No en vano, el origen de este tipo de instrumentos es la investigación en psicología del aprendizaje (Novak, 1984). Finalmente, hay que destacar una utilidad de los mapas conceptuales no directamente relacionada con este origen: su aplicación como sistema de presentación para conferencias o clases magistrales. Para este fin se añadieron a DigiDocMap algunas prestaciones adicionales de interactividad, como replegar y desplegar las ramas del árbol de conceptos, ocultar y mostrar zonas del mapa o cambiar los atributos tipográficos, como el tamaño o el tipo de letra. 
Tabla 2. Las tres versiones de DigiDocMap

\begin{tabular}{|c|c|c|c|}
\hline Característica & Versión 1 & Versión 2 & Versión 3 \\
\hline Fecha de creación & Septiembre 2002 & Septiembre 2004 & Abril 2005 \\
\hline Última actualización & Febrero 2003 & Julio 2004 & Abril 2005 \\
\hline Datos en formato & XTM (Topics Map) & $\begin{array}{l}\text { Array javascript } \\
\text { Array java }\end{array}$ & $\begin{array}{l}\text { Array javascrip } \\
\text { Array java }\end{array}$ \\
\hline Opciones importar & No & De la versión anterior & $\begin{array}{l}\text { De la versión anterior } \\
\text { De XML-Topic Map }\end{array}$ \\
\hline Opciones exportar & No & No & A Topic Map \\
\hline Multijerarquía & No & No & Sí \\
\hline $\begin{array}{l}\text { Posibilidad de inserción } \\
\text { de gráficos en los } \\
\text { conceptos }\end{array}$ & No & No & Sí \\
\hline Disposición de los mapas & Automática & Automática & Automática y manual \\
\hline Interactividad del mapas & - & $\begin{array}{l}\text { Desplegar/replegar } \\
\text { Resaltar/atenuar } \\
\text { Mostrar/ocultar menú }\end{array}$ & $\begin{array}{l}\text { Desplegar/replegar } \\
\text { Resaltar/atenuar } \\
\text { Mostrar/ocultar menú } \\
\text { Seleccionar y arrastrar }\end{array}$ \\
\hline $\begin{array}{l}\text { Modelo teórico de la } \\
\text { estructura }\end{array}$ & Árbol sin dirección & Árbol sin dirección & Grafo dirigido \\
\hline $\begin{array}{l}\text { Listado imprimible de } \\
\text { enlaces }\end{array}$ & Sí & Sí & Sí \\
\hline Guardar el mapa & $\begin{array}{l}\text { Copiando y pegando un } \\
\text { fragmento de código }\end{array}$ & Automático en C:/temp & $\begin{array}{l}\text { Automático en cualquier } \\
\text { disco y directorio }\end{array}$ \\
\hline Menú de configuración & No & Sí & Sí \\
\hline Referencias cruzadas & No & Parcialmente & Sí \\
\hline Orientación del mapa & Arriba-abajo & $\begin{array}{l}\text { Arriba-abajo } \\
\text { Izquierda-derecha }\end{array}$ & $\begin{array}{l}\text { Arriba-abajo } \\
\text { Izquierda-derecha } \\
\text { Cualquier otra } \\
\text { orientación } \\
\end{array}$ \\
\hline Definición de vistas & No & Sí & Sí \\
\hline $\begin{array}{l}\text { Uso libre para instalar } \\
\text { en local }\end{array}$ & No & Sí & Sí \\
\hline Uso libre en la Web & Sí & Sí & Sí \\
\hline $\begin{array}{l}\text { Lenguaje de } \\
\text { programación }\end{array}$ & javascript & javascript y java & javascript y java \\
\hline $\begin{array}{l}\text { Operativo en } \\
\text { navegadores }\end{array}$ & $\begin{array}{l}\text { Internet Explorer } 5.00 \\
\text { superior }\end{array}$ & $\begin{array}{l}\text { Internet Explorer } 5.00 \\
\text { superior } \\
\text { Mozilla } 5.0 \text { o superior }\end{array}$ & $\begin{array}{l}\text { Internet Explorer } 5.00 \\
\text { superior } \\
\text { Mozilla } 5.0 \text { o superior }\end{array}$ \\
\hline
\end{tabular}

\section{Versión 1 de DigiDocMap}

La primera versión del editor de mapas fue creada a finales del año 2002 y desarrollada enteramente en lenguaje javascript, y para guardar los mapas realizados el editor proporcionaba un fragmento código HTML y javascript (código de instalación) que el usuario copiaba y pegaba en una página HTML. Los 
mapas eran almacenados en formato XTM (Topics Map en XML) y el navegador procesaba directamente estos datos usando la prestación isla de datos, sólo disponible en el navegador Internet Explorer. En esta primera versión el editor era un servicio disponible en Internet, no podía instalarse en el ordenador local del usuario.
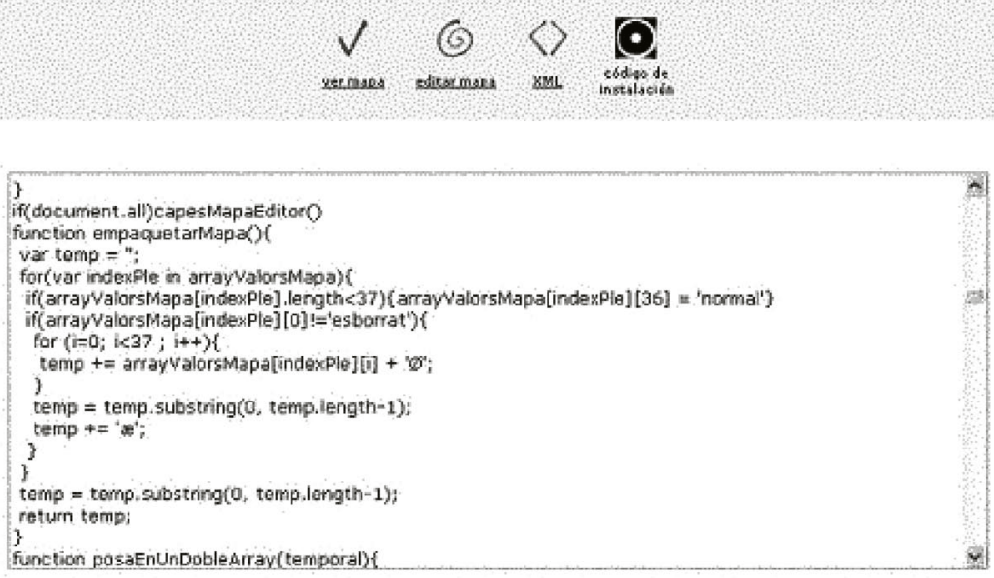

Fig. 2. Código de instalación para crear un mapa (DigiDocMap Ver 1.0)

\section{Versión 2 de DigiDocMap}

La segunda versión fue creada dos años después para incorporar mayores prestaciones de interactividad, acceder al disco local del usuario para guardar el mapa, mejorar la compatibilidad con distintos navegadores y permitir su instalación en el ordenador local.

Debido a los desajustes apuntados entre las características de los mapas conceptuales (Novak 1984) y el formato Topic Maps, se decidió usar el formato XTM exclusivamente para intercambiar datos entre aplicaciones por medio de opciones de importación y exportación. A partir de la versión dos, los datos fueron almacenados usando estructuras de datos de javascript y java más ajustadas a los requerimientos de los mapas conceptuales. Esta mejora hizo posible que los mapas creados pudieran visualizarse en cualquier navegador y se superaron las limitaciones de la primera versión.

Por otra parte, el editor recoge los datos que los usuarios introducen por medio de formularios y genera el mapa conceptual colocando cada elemento de forma automática. La disposición de cada elemento está determinada por el algoritmo correspondiente y el usuario puede decidir en qué orden pone los conceptos o frases de enlace pero no en qué coordenadas van a aparecer. 
Como veremos en la versión tres, estas limitaciones son superadas al incorporar herramientas que permiten seleccionar y arrastrar los elementos del mapa, tanto en el momento de su creación, como después, cuando el usuario interactúa con un mapa ya creado.

Otro cambio significativo de esta segunda versión fue su desarrollo en java lo que permitió grabar en el disco del usuario los mapas realizados. En la primera versión el editor era exclusivamente un servicio disponible en Internet, a partir de la segunda versión se ofreció además la posibilidad de instalar el editor en el ordenador del usuario como aplicación de uso libre (freeware).

Durante los dos años que se utilizó la primera versión se constató la potencialidad de los mapas conceptuales para hacer presentaciones en clases magistrales o conferencias. Para potenciar este nuevo e imprevisto uso se desarrollaron algunas prestaciones de interactividad. Por ejemplo se añadieron botones para que las distintas ramas del árbol pudieran replegarse y desplegarse, para resaltar o atenuar los conceptos e incluso para orientar el mapa de izquierda a derecha, en lugar de arriba a abajo como aparece por defecto.

En esta segunda versión se incorporó por primera vez un menú en la parte superior de los mapas para controlar su presentación y poder modificar los elementos de interactividad, la tipografía y otras características, como la presencia del propio menú.

El listado de enlaces es una característica del editor ya presente desde la primera versión y que fue la respuesta a un problema detectado cuando un mapa se imprimía. Cuando un concepto tiene recursos asociados aparece un enlace que permite abrir una ventana adicional, el listado de las URL (Uniform Resource Lorato, Localizador Uniforme de Recursos LUR) hacia estos recursos. Esta forma de implementar la navegación tiene la ventaja de poder incorporar gran cantidad de enlaces en un mapa sin que se ello provoque una sobrecarga de información en poco espacio. El usuario abrirá sólo los que le interese consultar en un momento dado. El inconveniente de trabajar con ventanas adicionales es que la impresión del mapa no incluye la información de los enlaces. El problema se solucionó incorporándole al mapa un listado global de todos los enlaces. 

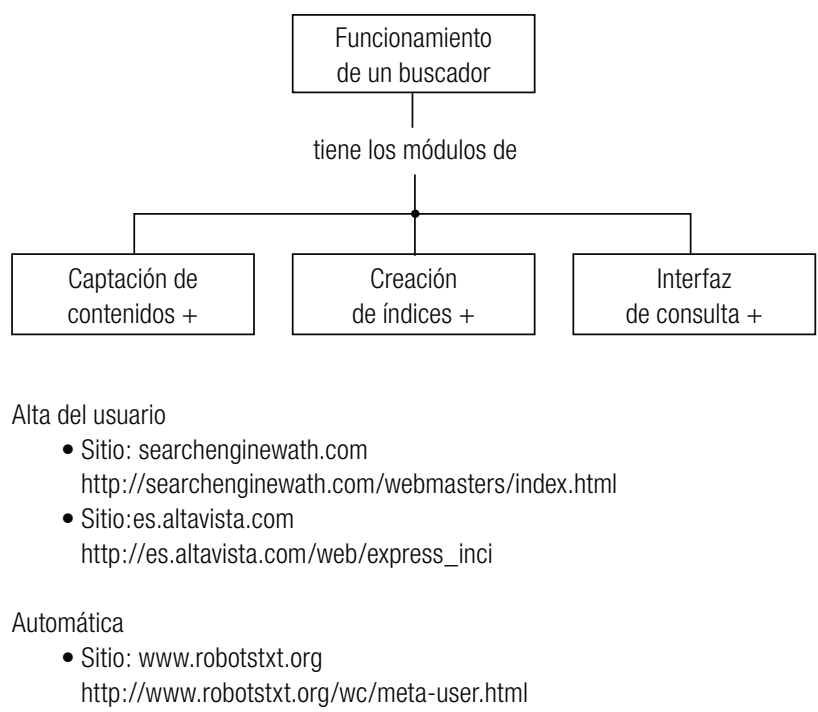

Fig. 3. Listado de enlaces de un mapa (DigiDocMap Ver 2.0)

La versión dos tuvo como novedad importante la definición de vistas. Una vista es una de las posibles formas de mostrar un mapa en función de la tipografía, atenuación, replegado y orientación. De manera que un mapa puede tener infinitas vistas según la elección que hagamos de cada uno de estos elementos. El editor permite grabar con un nombre este conjunto de características para luego aplicarlas de golpe al mapa. El menú superior tiene un listado desplegable con las vistas definidas.

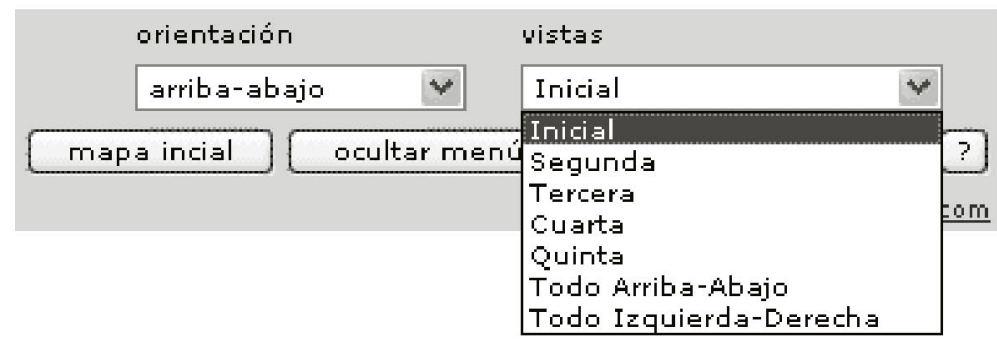

Fig. 4. Listado desplegable para seleccionar una vista (DigiDocMap Ver 2.0)

Novak aconseja incorporar referencias cruzadas para relacionar diferentes dominios del conocimiento presentes en el mapa conceptual. En la práctica crear una referencia cruzada implica relacionar conceptos en ramas distintas de la estructura del árbol de conceptos. Con la presencia de referencias 
cruzadas aumentaba exponencialmente la complejidad del algoritmo para la colocación automática de los elementos del mapa, por lo que en la segunda versión sólo fueron soportadas parcialmente.

\section{Versión 3 de DigiDocMap}

Dos factores fueron determinantes para tomar la decisión de desarrollar una nueva versión del editor DigiDocMap: la necesidad de crear opciones de exportación a XTM (LEM) y el deseo de superar las limitaciones relativas a las referencias cruzadas. Aunque este segundo factor pueda parecer inocente, determinó un cambio radical en la configuración interna del mapa y evolucionó desde la estructura de árbol hasta la de grafo.

En las dos primeras versiones del editor los mapas obedecían a la estructura interna de un árbol, de manera que partiendo de un nodo inicial (concepto raíz) el mapa se ramificaba mediante frases de enlace y nuevos conceptos. No era posible crear relaciones de multijerarquía, ni establecer plenamente referencias cruzadas entre conceptos de diferentes ramas.

Para superar esta limitación se reconstruyeron completamente los algoritmos relativos a la colocación automática de los elementos del mapa y se rompieron así las limitaciones de la estructura en árbol al añadir la polijerarquía. Además se añadió la posibilidad de colocación manual de los componentes del mapa con selección y desplazamiento, y se estableció un proceso mixto automático-manual para crear su aspecto final. Se mejoró la representación de la relaciones incorporando flechas para indicar, en caso necesario, la dirección de la frase de enlace, y se añadió la posibilidad de insertar gráficos en los mapas como ilustraciones de los conceptos.

En lo relativo a las opciones de importación-exportación, la tercera versión incorporó la posibilidad de usar el formato XTM (LEM) para intercambiar datos usando la concreción de la norma realizada por CmapTools

http://cmap.ihmc.us/.

\section{Conclusiones}

El desarrollo del editor de mapas conceptuales DigiDocMap es un proyecto que se está desarrollando desde el año 2002. En este artículo se describe el desarrollo de las tres versiones creadas de la aplicación. Los próximos objetivos de este proyecto de investigación son incorporarle al editor herramientas que permitan ayudarles a los autores en la creación de los mapas sugiriéndoles nuevos conceptos o evaluando la adecuación de las frases de enlace. 


\section{BiBLIOGRAFÍA}

D.P. Ausubel; J.D. Novak, H. Hanesian. Psicología educativa. Mexico: Trillas, 1989.

Noemi Betancort Cabrera; Lidia Chozas Mahillo Tesauros, "Mapas Conceptuales y Topic Maps".

http://es.geocities.com/naolig/tesauros-mapas-conceptuales-topic-maps.htm [Consulta: 15/03/2005]

Buzan, T. The Mind Map Book, New York: Penguin, 1991.

Michel Biezunski, "Understanding Topic Maps”, 2005. http://www.infoloom.com/whitepaper.htm [Consulta:3/03/2005]

CmapTools http://cmap.ihmc.us/ [Consulta: 03/03/2005]

Lluís Codina, "Posicionamiento Web: Conceptos y Ciclo de Vida", Hipertext.net, núm. 2, 2004. http://www.hipertext.net [Consulta:3/03/2005]

Lluís Codina, "La web semántica: una visión crítica", en El profesional de la información, vol. 12, núm. 2, 2003.

Lluís Codina, "Internet invisible y web semántica: ¿el futuro de los sistemas de información en línea”, en Tradumática, núm. 2, 2003.

Juan C. Dürsteler. “Conceptual Maps”, 2004-03-01 http://www.infovis.net/printMag.php?num=141\&lang=2 [Consulta: 3/03/2005]

Yusef Hassan Montero, "Diseño de Arquitecturas de Información: Descripción y Clasificación”, http://www.nosolousabilidad.com/articulos/descripcion_y_clasificacion.htm [Consulta: 15/03/2005]

Lars Marius Garshol, "Metadata? Thesuari? Taxonomies? Topic Maps! Making sense of it all”, 2004.

http:/www.ontopia.net/topicmaps/materials/tm-vs-thesauri.html [consulta 03/03/2005]

Lars Marius Garshol, "What Are Topic Maps?, September 11, 2002. http://www.xml.com/pub/a/2002/09/11/topicmaps.html [Consulta: 3/03/2005]

J. D. Novak, Teoría y práctica de la educación, Madrid: Alianza, 1982.

J. D. Novak; D. B. Gowin, Learning How to Learn, New York and Cambridge, UK: Cambridge University Press, 1984.

J. D. Novak; D. B. Gowin, Aprendiendo a aprender, Barcelona: Martínez Roca, 1988.

ISO/IEC 13250:2000 “Topic Maps”, 19 may 2002. http://www.y12.doe.gov/sgml/sc34/document/0322_files/iso132502nd-ed-v2.pdf [Consulta:3/03/2005]

Jan Lanzing, “The Concept Mapping Homepage”, [1997]. http://users.edte.utwente.nl/lanzing/cm_home.htm [Consulta: 03/03/2005]

Marcos Mora, Mari Carmen, Interacción en interfaces de recuperación de información: conceptos, metáforas y visualización, Gijón: Trea, 2004. 
Master Online en Documentación Digital. http://www.documentaciondigital.org [Consulta: 03/03/2005]

J. A. Moreiro; S. Sánchez Cuadrado; J. Morato, "Panorámica y tendencias en topic maps”, Hipertext.net, núm. 1, 2003. http://www.hipertext.net [Consulta: 04/03/2005].

J. A. Moreiro et al, "De los tesauros a los Topic Maps: nuevo estándar para la representación y la organización de la información”, Encuentros Bibli: Revista electronica de Biblioteconomia e Ciencias da informaçao. Núm. 18-2, 2004.

A. Notoria, Mapas conceptuales una técnica para aprender, Madrid: Narcea, 1992.

Joseph D. Novak, "The Theory Underlying Concept Maps and How To Construct Them", http://cmap.coginst.uwf.edu/info/printer.html [Consulta:3/03/2005]

Steve Pepper, "The TAO of Topic Maps finding the way in the age of infoglut",

http://www.ontopia.net/topicmaps/materials/tao.html

[Consulta: 15/032005]

Cristòfol Rovira, El disseny de navegació i l'organtizació de continguts en l'hipertext per a l'ensenyament - aprenentatge, Tesis Doctoral, Universitat de Barcelona, 2001.

Cristòfol Rovira, "Mapas conceptuales para la representación del conocimiento", en Cristòfol Rovira; Lluís Codina (dir.). Documentación digital. Barcelona: Sección Científica de Ciencias de la Documentación, Departamento de Ciencias Políticas y Sociales, Universidad Pompeu Fabra, 2003.

http://www.documentaciondigital.org [Consulta:3/03/2005]

Cristòfol Rovira, Lluís Codina, "La orientación a objetos en el diseño de sedes web: hipertextos y representación de la información", en Revista española de documentación científica, Vol 26, núm. 3, 2003

Rovira, Cristòfol; Codina, Lluís; Marcos, Mari Carmen; Palma, María del Valle, Información y documentación digital. Barcelona: IULA; Documenta Universitaria, 2004.

Techquila.

http://www.techquila.com/topicmaps.html [Consulta:3/03/2005]

XML Topic Maps (XTM) 1.0. 2001.

http://www.topicmaps.org/xtm/1.0/ [Consulta:3/03/2005] 